\title{
Cerebral Small Vessel Disease: Cognition, Mood, Daily Functioning, and Imaging Findings from a Small Pilot Sample
}

\author{
John G. Baker ${ }^{a}$ Amy J. Williams ${ }^{a}$ Catalina C. Ionita ${ }^{b}$ \\ Peterkin Lee-Kwen ${ }^{b}$ Marilou Ching ${ }^{b}$ Robert S. Miletich ${ }^{a, b}$ \\ Departments of a Nuclear Medicine and ${ }^{\mathrm{b}}$ Neurology, State University of New York at Buffalo, \\ Buffalo, N.Y., USA
}

\section{Key Words}

Cerebrovascular disease $\cdot$ Cerebral small vessel disease $\cdot$ Stroke $\cdot$ SPECT $\cdot$ Neuropsychology Subcortical vascular cognitive impairment $\cdot$ CogHealth $\cdot$ RBANS $\cdot$ SF-36

\begin{abstract}
Cerebral small vessel disease, a leading cause of cognitive decline, is considered a relatively homogeneous disease process, and it can co-occur with Alzheimer's disease. Clinical reports of magnetic resonance imaging (MRI)/computed tomography and single photon emission computed tomography (SPECT) imaging and neuropsychology testing for a small pilot sample of 14 patients are presented to illustrate disease characteristics through findings from structural and functional imaging and cognitive assessment. Participants showed some decreases in executive functioning, attention, processing speed, and memory retrieval, consistent with previous literature. An older subgroup showed lower age-corrected scores at a single time point compared to younger participants. Performance on a computer-administered cognitive measure showed a slight overall decline over a period of 8-28 months. For a case study with mild neuropsychology findings, the MRI report was normal while the SPECT report identified perfusion abnormalities. Future research can test whether advances in imaging analysis allow for identification of cerebral small vessel disease before changes are detected in cognition.
\end{abstract}




\section{Introduction}

As our life span lengthens, and as the proportion of older adults in our population grows, cognitive changes from cerebrovascular disease are becoming an increasingly common problem. With disease progression these changes can result in dementia, with decreased quality of life and a substantial burden of care for family members and the larger community.

While Alzheimer's disease is the most common cause of dementia, cerebrovascular disease has been considered the second most common etiology of dementia in the US [1]. Cerebral small vessel disease, as distinct from large vessel disease and other forms of cerebrovascular disease, is considered the most common etiology of vascular dementia. As noted in Pantoni [2], 'The term cerebral small vessel disease refers to a group of pathological processes with various aetiologies that affect the small arteries, arterioles, venules, and capillaries of the brain. ... Because lacunar infarcts and white matter lesions are easily detected by neuroimaging, whereas small vessels are not, the term small vessel disease is frequently used to describe the parenchyma lesions rather than the underlying small vessel alterations'.

It is estimated that older adults with small vessel disease develop vascular dementia between 5 and 25 times more often than age-matched general population samples [1]. In clinical studies, estimates of the proportion of dementia caused by small vessel disease range from 36 to $67 \%$ [3]. In one study, mild cognitive impairment (MCI) preceded subcortical small vessel dementia in a substantial proportion of individuals [4].

The clinical diagnostic work-up of cognitive impairment from cerebrovascular disease and other etiologies generally includes neuropsychology testing to identify patterns of impairment and often neuroimaging to aid in determining disease processes. Cognitive changes from cerebral small vessel disease generally include decreases in attention and working memory, processing speed, executive functioning, and aspects of memory performance i.e., primarily retrieval and encoding rather than retention [5-9]. Zhou and Jia [10] found that patients with MCI from small vessel disease performed worse on processing speed and better on memory than those with MCI from Alzheimer's disease. Of course, cerebrovascular disease can occur with Alzheimer's disease in so-called 'mixed' dementia.

Brief, repeatable neuropsychological measures, such as the Repeatable Battery for Assessment of Neuropsychological Status (RBANS), have been developed that are well suited to assess and monitor cognition among older adults. Age- and education-adjusted normative data for the RBANS are available for community-dwelling individuals between 65 and 89 [11], and regression-based formulas for predicting change in scores have been developed for this same age group [12].

Computer-administered, very brief, repeatable assessments of cognitive performance, such as the CogHealth program, may also assist with identifying MCI and monitoring its progression with minimal practice effects. Darby et al. [13] found a sensitivity of $80 \%$ and a specificity of $95 \%$ for CogHealth in differentiating normal from MCI participants with multiple administrations at a single time point. Normal control and MCI groups were also different on change in memory performance from baseline to a 12-month follow-up. Four scales of the CogHealth battery measuring attention functions, processing speed, working memory, and continuous visual recognition learning correlated with six traditional neuropsychological measures (Spatial Span, Symbol Digit Modalities, Grooved Pegboard, Trail Making Test, Brief Visual Memory Test Total Immediate Recall, and Rey Complex Figure 30 Minute Recall) to a degree that provided support for the construct validity of specific scales of the computer measure [14].

Both structural neuroimaging, including magnetic resonance imaging (MRI) and computed tomography $(\mathrm{CT})$, and functional neuroimaging, including single photon emission 
computed tomography (SPECT) and positron emission tomography (PET), have been used to determine disease processes related to cognitive decline. Sabri et al. [15] compared evidence of cerebral small vessel disease from PET, SPECT, and MRI to cognitive impairment. They found that neuropsychological deficits correlated well with decreased glucose metabolism on PET and decreased regional cerebral blood flow on SPECT. Lacunar infarctions and deep white matter hyperintensities seen on MRI did not correlate with neuropsychological deficits nor PET metabolism and SPECT blood flow. They concluded that lacunar infarctions and deep white matter hyperintensities seen on MRI may be epiphenomena that morphologically characterize cerebral small vessel disease, but do not in and of themselves indicate cognitive impairment. Yoshikawa et al. [16] suggested that three-dimensional assessment of cerebral blood flow with SPECT may be able to detect incipient dementia in clinical settings before morphologic changes are seen on MRI or CT.

Nonetheless, a more recent study found that periventricular white matter lesions, lacunar infarcts, and atrophy on MRI were specifically associated with decline in information processing speed and executive function [9]. Grau-Olivares et al. [17] compared neuropsychology test scores across five subtypes of first-ever acute lacunar infarction on MRI and found that mild neuropsychological disturbances occurred in more than half the cases, with $55 \%$ fulfilling the criteria for MCI of vascular origin. In a recent review of the literature on MCI in patients with small vessel disease, Grau-Olivares and Arboix [5] note that neuropsychological abnormalities from small vessel disease occur more frequently than believed previously, and that patients with subcortical vascular dementia represent a highly prevalent and homogeneous group.

Authors have noted that subcortical ischemic vascular dementia from small vessel disease has been underrecognized in clinical settings and understudied in the research literature $[5,17]$. In this pilot study, we present some preliminary cognitive, mood, daily functioning, and imaging data from a small pilot sample of patients with cerebral small vessel disease. Age-corrected cross-sectional test scores are compared between older and younger groups, and we present multiple computerized test scores over a 1- to 3-year period. We include a case study to illustrate the relation between neuropsychological test results and findings from clinical reports of structural and functional imaging. We hypothesized that we would find decreases in neuropsychological measures characteristic of cerebral small vessel disease (i.e. attention, speed, executive function, and memory retrieval) with greater decreases among older participants. We also expected to find heterogeneous regions of hypoperfusion in SPECT reports for individual participants in the pilot sample.

\section{Methods}

\section{Participants}

Patients seen for follow-up at a stroke center clinic were screened according to specific inclusion and exclusion criteria as specified on a checklist used by the neurologists and research nurses who recruited patients for this study. Patients were eligible if they were $>45$ years, had a diagnosis of hypertension or diabetes, and at least one other vascular risk factor.

To be included patients also needed to be determined to be at risk for subcortical vascular cognitive impairment [as specified in 18-20]. Examples included a history of subcortical stroke; dizziness due to hypoperfusion; gait disturbance or other motor dysfunction (e.g. subtle focal signs such as arm drift, unilateral incoordination, extrapyramidal features); reported cognitive impairment such as dysexecutive syndrome or forgetfulness; or neuroimaging findings such as white matter hyperintensities or lacunes. 
Dement Geriatr Cogn Disord Extra 2012;2:169-179

DOI: 10.1159/000333482

Published online: April 18, 2012

2012 S. Karger AG, Base

www.karger.com/dee

Baker et al.: Cerebral Small Vessel Disease

Exclusion criteria included significant known large vessel cerebrovascular disease; a history of large vessel stroke or large vessel transient ischemic attack; a current diagnosis of dementia; use of cholinesterase inhibitors or other dementia medications; a pattern of symptoms consistent with Alzheimer's disease or other neurodegenerative disease etiologies that could lead to MCI or dementia (e.g. Parkinson's Disease, Multiple Sclerosis, Traumatic Brain Injury); or a life expectancy of less than a year.

As noted above, with the exception of one participant, patients were recruited from a stroke center clinic, and generally had experienced a mild clinically evident lacunar stroke. The study was explained to eligible patients, and interested patients read and signed an informed consent form. The study was approved by the University at Buffalo Health Sciences Institutional Review Board.

\section{Procedures}

Measures of Cognition, Mood, and Daily Functioning

Study participants were administered an initial assessment that included traditional and computer-based measures, and follow-up assessments with only the computer-based assessment and brief mood and daily functioning questionnaires. At initial assessment, participants completed a short battery of neuropsychological tasks including the RBANS, the Digit Span subtest of the Wechsler Adult Intelligence Scale-Third Edition (WAIS), Trail Making Test - Parts A and B (TMT A, TMT B), Clock Drawing Tests (CLOX1, CLOX2), Controlled Oral Word Association Test (FAS), Animals Test of Semantic Verbal Fluency (Animals), Geriatric Depression Scale (GDS), Medical Outcomes Study Short Form-36 (SF-36), and the CogHealth Memory Monitoring System (CogHealth). At the follow-up assessments, participants completed only the CogHealth, GDS, and SF-36.

\section{Neuroimaging}

Participants received a SPECT imaging study at the time of their enrollment using Neurolite. These imaging studies were read by a dual board certified neurologist and nuclear medicine physician and a clinical report was generated. Clinical reports from other imaging studies completed before and during the period of the study (i.e. existing MRI or CT images as part of stroke assessment or other regular clinical care) were included as part of the study.

\section{Results}

Fourteen patients who met the inclusion and exclusion criteria enrolled in the study. Table 1 presents demographics for this pilot sample. As indicated in table 1, participants are predominantly female and White, and many have some college education. There is a wide age range from 50 to 86 years. The sample was divided into an older and younger group based on the median age.

Table 2 presents mean standard scores for the cognitive, mood, and daily functioning measures for the older, younger, and combined age groups at the initial assessment. As can be seen in table 2, the age-referenced index scores from the RBANS manual and the agereferenced subtests scores from Lezak et al. [21], as well as the norm-referenced scores for other traditional neuropsychological tests are generally somewhat lower for the older group. These differences reached statistical significance for the RBANS story memory and coding subtests and for the GDS raw scores. Differences for the RBAN3 Immediate Memory Index score approached significance.

While many of the mean scores for the younger group are close to the mean for their age groups (i.e. a standard score of 100 and a standard deviation of 15), many of the mean scores 
Table 1. Demographic information

\begin{tabular}{|c|c|c|c|}
\hline & $\begin{array}{l}\text { Younger participants } \\
\text { (ages 50-71) }\end{array}$ & $\begin{array}{l}\text { Older participants } \\
\text { (ages } 72-86 \text { ) }\end{array}$ & Entire sample \\
\hline Number of participants & 7 & 7 & 14 \\
\hline Mean age $\pm S D$, years & $61.7 \pm 7.65$ & $78.3 \pm 5.18$ & $70.0 \pm 10.65$ \\
\hline Mean education $\pm S D$, years & $14.9 \pm 2.04$ & $13.0 \pm 3.56$ & $13.93 \pm 2.95$ \\
\hline Sex, \% female & 57.1 & 71.4 & 64.3 \\
\hline Ethnicity, \% White & 85.7 & 100.0 & 92.9 \\
\hline
\end{tabular}

Table 2. RBANS index scores and subtest scores (age-corrected standard scores as defined by RBANS manual), TMT A and B standard scores, CLOX1 and 2 standard scores, FAS standard score, WAIS Digit Span subtest standard scores, SF-36 daily functioning scores, and GDS raw scores for baseline sample

\begin{tabular}{|c|c|c|c|c|c|c|}
\hline \multirow[t]{2}{*}{ Measure } & \multicolumn{2}{|c|}{$\begin{array}{l}\text { Younger participants } \\
\text { (ages 50-71) } \\
(\mathrm{n}=7)\end{array}$} & \multicolumn{2}{|c|}{$\begin{array}{l}\text { Older participants } \\
\text { (ages } 72-86) \\
(\mathrm{n}=7)\end{array}$} & \multicolumn{2}{|c|}{$\begin{array}{l}\text { Combined sample means } \\
\text { and group difference effect } \\
\text { sizes (ages } 50-86)(n=14)\end{array}$} \\
\hline & mean $\pm \mathrm{SD}$ & range & mean $\pm \mathrm{SD}$ & range & mean $\pm \mathrm{SD}$ & effect size \\
\hline \multicolumn{7}{|l|}{ RBANS index and subtest scores } \\
\hline Immediate memory & $110 \pm 8.4$ & $94-120$ & $91 \pm 24.7^{\mathrm{a}}$ & $53-117$ & $101 \pm 20.4$ & 0.28 \\
\hline List learning & $110 \pm 12.2$ & $84-120$ & $94 \pm 25.6$ & $57-123$ & $102 \pm 21.0$ & 0.21 \\
\hline Story memory & $111 \pm 7.6$ & $98-118$ & $88 \pm 21.4^{\mathrm{b}}$ & $57-103$ & $99 \pm 19.7$ & 0.39 \\
\hline Visuospatial/constructional & $111 \pm 15.0$ & $92-131$ & $107 \pm 6.6$ & $96-116$ & $109 \pm 11.3$ & 0.08 \\
\hline Figure copy & $105 \pm 22.5$ & $55-119$ & $106 \pm 12.7$ & $83-118$ & $105 \pm 17.5$ & -0.01 \\
\hline Line orientation & $108 \pm 11.2$ & $88-119$ & $107 \pm 9.0$ & $93-114$ & $108 \pm 9.8$ & 0.04 \\
\hline Language & $100 \pm 9.2$ & $88-113$ & $100 \pm 9.5$ & $85-112$ & $100 \pm 9.0$ & 0.08 \\
\hline Picture naming & $102 \pm 8.7$ & $91-109$ & $101 \pm 11.0$ & $84-109$ & $101 \pm 9.6$ & 0.04 \\
\hline Semantic fluency & $101 \pm 14.8$ & $80-121$ & $103 \pm 21.4$ & $72-139$ & $102 \pm 17.7$ & -0.03 \\
\hline Attention & $102 \pm 8.4$ & $88-115$ & $94 \pm 11.8$ & $79-109$ & $98 \pm 10.8$ & 0.22 \\
\hline Digit span & $104 \pm 16.4$ & $86-134$ & $101 \pm 16.1$ & $82-128$ & $102 \pm 15.7$ & 0.06 \\
\hline Coding & $101 \pm 7.0$ & $92-110$ & $87 \pm 11.7^{\mathrm{c}}$ & $68-105$ & $94 \pm 11.6$ & 0.39 \\
\hline Delayed memory & $101 \pm 9.9$ & $81-112$ & $94 \pm 22.8$ & $48-114$ & $98 \pm 17.3$ & 0.11 \\
\hline List recall & $106 \pm 13.5$ & $93-129$ & $104 \pm 17.0$ & $71-127$ & $105 \pm 14.8$ & 0.03 \\
\hline List recognition & $103 \pm 9.0$ & $85-108$ & $93 \pm 22.1$ & $48-113$ & $98 \pm 16.9$ & 0.15 \\
\hline Story recall & $101 \pm 12.7$ & $84-119$ & $95 \pm 23.7$ & $52-114$ & $98 \pm 18.5$ & 0.07 \\
\hline Figure recall & $98 \pm 10.2$ & $84-117$ & $89 \pm 15.4$ & $59-106$ & $94 \pm 13.5$ & 0.20 \\
\hline Total score & $106 \pm 9.2$ & $89-117$ & $96 \pm 14.8$ & $70-110$ & $101 \pm 13.1$ & 0.23 \\
\hline TMT - Part A $\mathrm{A}^{\mathrm{e}}$ & $106 \pm 9.6$ & $97-123$ & $96 \pm 9.3$ & $87-109$ & $101 \pm 10.4$ & 0.25 \\
\hline TMT - Part B ${ }^{f}$ & $99 \pm 21.1$ & $60-118$ & $94 \pm 7.3$ & $82-101$ & $97 \pm 15.9$ & 0.08 \\
\hline $\mathrm{CLOX}^{\mathrm{e}}$ & $109 \pm 7.4$ & $95-117$ & $103 \pm 11.8$ & $95-117$ & $106 \pm 9.6$ & 0.13 \\
\hline $\mathrm{CLOX} 2^{\mathrm{f}}$ & $104 \pm 7.9$ & $97-112$ & $109 \pm 5.4$ & $101-112$ & $106 \pm 7.2$ & -0.17 \\
\hline FAS $^{\mathrm{e}}$ & $97 \pm 11.0$ & $86-112$ & $91 \pm 15.0$ & $75-106$ & $94 \pm 12.7$ & 0.12 \\
\hline WAIS Digit Span subtest ${ }^{g}$ & $104 \pm 9.7$ & $90-120$ & $94 \pm 7.5$ & $85-100$ & $100 \pm 10.0$ & 0.26 \\
\hline GDS & $13 \pm 7.0$ & $4-21$ & $6.4 \pm 4.5^{\mathrm{d}}$ & $0-13$ & $9.9 \pm 6.7$ & 1.07 \\
\hline \multicolumn{7}{|l|}{ SF-36 } \\
\hline Physical composite & $87 \pm 16.8$ & $56-106$ & $87 \pm 17.7$ & $58-106$ & $87 \pm 16.6$ & 0.01 \\
\hline Mental (emotional) composite & $84 \pm 16.9$ & $55-100$ & $100 \pm 21.0$ & $69-125$ & $92 \pm 20.2$ & -0.24 \\
\hline
\end{tabular}


Table 3. Comparison of CogHealth performance at baseline to follow-up

\begin{tabular}{|c|c|c|c|c|c|c|}
\hline \multirow[t]{2}{*}{ Measure } & \multicolumn{2}{|l|}{$\begin{array}{l}\text { Baseline } \\
(\mathrm{n}=9)\end{array}$} & \multicolumn{2}{|c|}{$\begin{array}{l}\text { Follow-up ( } 8 \text { months- } \\
28 \text { months })(n=9)\end{array}$} & \multicolumn{2}{|c|}{$\begin{array}{l}\text { Paired difference } \\
\text { and effect size }\end{array}$} \\
\hline & mean $\pm \mathrm{SD}$ & range & mean $\pm \mathrm{SD}$ & range & mean $\pm \mathrm{SD}$ & effect size \\
\hline \multicolumn{7}{|l|}{ CogHealth } \\
\hline Detection 1: speed of processing & $101.6 \pm 9.5$ & $85-108$ & $97.6 \pm 16.6$ & $67-108$ & $4.0 \pm 9.1$ & 0.15 \\
\hline $\begin{array}{l}\text { Identification: visual identification task } \\
\text { One back: working memory and }\end{array}$ & $101.1 \pm 6.1$ & $88-107$ & $100.0 \pm 8.5$ & $80-107$ & $1.1 \pm 10.2$ & 0.07 \\
\hline executive functioning & $99.9 \pm 9.9$ & $78-115$ & $96.7 \pm 5.8$ & $90-106$ & $3.2 \pm 9.2$ & 0.19 \\
\hline One card learning: card-based & & & & & & \\
\hline episodic memory and attention task & $102.0 \pm 9.0$ & $93-111$ & $101.3 \pm 8.7$ & $88-111$ & $0.7 \pm 7.0$ & 0.04 \\
\hline Monitoring: divided and spatial attention & $98.0 \pm 8.1$ & $84-106$ & $96.7 \pm 7.0$ & $85-105$ & $1.3 \pm 9.2$ & 0.09 \\
\hline $\begin{array}{l}\text { Associate Learning: contextual memory } \\
\text { and learning }\end{array}$ & $98.6 \pm 7.0$ & $86-109$ & $97.1 \pm 6.7$ & $88-108$ & $1.4 \pm 5.8$ & 0.11 \\
\hline \multicolumn{7}{|l|}{ SF-36 $(n=8)$} \\
\hline Physical composite (PCS) & $85.2 \pm 20.4$ & $56-106$ & $87.3 \pm 16.8$ & $60-106$ & $-2.0 \pm 15.0$ & -0.06 \\
\hline Mental (emotional) composite (MCS) & $98.9 \pm 17.2$ & $69-125$ & $99.4 \pm 16.2$ & $69-114$ & $-0.5 \pm 7.7$ & -0.01 \\
\hline $\mathrm{GDS}^{\mathrm{a}}$ & $8.1 \pm 6.8$ & $0-18$ & $6.6 \pm 5.4$ & $0-16$ & $1.6 \pm 4.1$ & 0.12 \\
\hline
\end{tabular}

${ }^{\text {a }}$ GDS is a raw score where the score is equal to the number of depressive symptoms endorsed.

Paired sample t tests did not show any statistically significant differences between baseline and follow-up scores.

Table 4. Clinical report findings for structural and functional neuroimaging and neuropsychology test scores below one standard deviation for pilot sample

\begin{tabular}{|c|c|c|c|c|}
\hline Case & Age group & $\begin{array}{l}\text { MRI: WMH/ } \\
\text { lacunes/ } \\
\text { SVD findings }\end{array}$ & $\begin{array}{l}\text { SPECT: } \\
\text { small vessel disease - } \\
\text { hypoperfusion }\end{array}$ & $\begin{array}{l}\text { Neuropsychological testing: domains below } 1 \text { SD from } \\
\text { the normative mean }\end{array}$ \\
\hline 1 & older & yes/yes/no & mild - moderate & Attn, Del Mem ${ }^{\mathrm{c}}$ \\
\hline 2 & older & yes/yes/yes & moderate & None $^{c}$ \\
\hline 3 & older & no/no/no & mild - moderate & Attn, Speed ${ }^{c}$ \\
\hline 4 & younger & no/yes/yes & mild - moderate & Del Mem, GDS, MCS \\
\hline 5 & older & yes/yes/yes & mild - moderate & Attn, Im and Del Mem, Lang, Ex Fx, GDS, MCS, PCS \\
\hline 6 & younger & no/no/yes & & Attn, Im and Del Mem, Lang, Ex Fx, GDS, PCS \\
\hline 7 & younger & yes/no/no & mild - moderate & GDS, MCS, PCS \\
\hline 8 & younger & yes/no/no & mild - moderate & Wk Mem, Ex Fx \\
\hline 9 & older & yes/no/yes & moderate & Im Mem, PCS \\
\hline 10 & younger & yes/no/yes & mild - moderate & GDS, MCS, PCS \\
\hline 11 & younger & yes/no/no & mild - moderate & VS/Const \\
\hline 12 & older & no/no/yes ${ }^{\mathrm{a}}$ & & $\begin{array}{l}\text { Attn, Wk Mem, Im and Del Mem, Lang, Ex Fx, MCS, } \\
\text { PCS }\end{array}$ \\
\hline 13 & younger & $\mathrm{no} / \mathrm{no} / \mathrm{no}^{\mathrm{b}}$ & incipient & Wk Mem, Ex Fx, MCS, PCS \\
\hline 14 & older & no/no/yes & mild & Attn, Wk Mem, Im Mem, Lang, VS/Const, Ex Fx \\
\hline
\end{tabular}

${ }^{a}$ Findings are consistent with cerebral amyloid angiopathy. ${ }^{b}$ CT Scan. ${ }^{c}$ Tests of executive functioning were not administered.

$\mathrm{WMH}=$ white matter hyperintensities; SVD = small vessel disease; Attn = Attention; Wk Mem = working memory; Im Mem = immediate memory; Del Mem = delayed memory; Speed = processing speed; Lang = language; VS/Const = visual spatial/constructional; Ex Fx = executive functioning; PCS = SF-36 physical composite summary score; MCS = SF-36 mental/emotional composite summary score. 
for the older group are somewhat below the mean score for their age group. The older group showed significantly less depression than the younger group as indicated by their raw scores on the GDS. Their scores on the SF-36 Mental Composite Scale (MCS), which are non-agereferenced to the general US population, also showed better mood and emotional role functioning compared to the younger group. Physical functioning (SF-36 Physical Composite Scale (PCS)) is about the same for the older and younger groups, based on non-age-referenced general US population norms. Since age effects on cognition are somewhat controlled for by the age-referenced norms, these cross-sectional results appear to indicate some degree of decrease in cognitive performance, but not mood or physical functioning, for the older group within this pilot sample of individuals with cerebral small vessel disease.

For the 10 participants over the age of 65 , standard scores were calculated for the RBANS index scores using both the age-referenced manual norms and the age- and education-referenced norms from Duff et al. [11]. While the RBANS total scale scores were similar using both norms, the RBANS individual index scores varied according to which norms were used. Attention, immediate memory, and delayed memory index scores were higher using the norms from Duff et al. [11], while language and visuospatial/construction were lower.

Table 3 presents scores for a subsample of 9 participants with available data for two time points using the computer-based cognitive measure, and the mood and daily functioning measures. For this subsample, the cognitive scores show a very slight and statistically nonsignificant decline over time, while the physical functioning and mood scores show a slight improvement.

Table 4 presents a summary of the clinical report findings for structural and functional imaging and the neuropsychology test result findings for the 14 participants. The presence and extent of small vessel disease is noted for each participant's available structural and functional imaging. It is important to note that although we used strict inclusion and exclusion criteria for cerebral small vessel disease, 9 of the 14 participants had questions of other disease processes raised in the clinical reports of functional and/or structural imaging. Of the 13 participants with neuropsychology test domains that are below one standard deviation from the normative mean score, 10 participants' scores include at least one domain that would be expected to be affected by cerebral small vessel disease (i.e. attention, speed, executive function, and memory retrieval).

A case study illustrates differences in structural and functional imaging findings with mild neuropsychology findings (please refer to Case 3 inside table 4). For this 84-year-old White woman, the neuropsychology test results, presented in figure 1, show some mild decreased performance on measures of executive function (CLOX1, FAS, TMT B), speed (TMT A), working memory (WAIS Digit span), and on the attention index score of the RBANS. No depression (GDS) or decreased daily functioning (PCS, MCS) was reported, beyond difficulty with managing a large house. While the decreases in cognitive performance are mild, they include areas typically affected by cerebral small vessel disease.

The MRI report for this case study described no significant intracranial abnormality, and no abnormality of perfusion. Nonetheless, the SPECT imaging report noted a mild to moderate multifocal pattern of hypoperfusion in the white matter, patchy hypoperfusion in the left lenticular nucleus, and regional hypoperfusion in the anterior temporal cortices (trace to mild) with some generalized increased heterogeneity of cortical perfusion bihemispherically, all consistent with small vessel disease. No pattern of hypoperfusion on SPECT was seen that would be suggestive of large vessel disease, neurodegenerative disease, or psychiatric disorder. This case illustrates the potential usefulness of functional imaging in identifying changes in perfusion in the absence of structural abnormalities identified on MRI. 


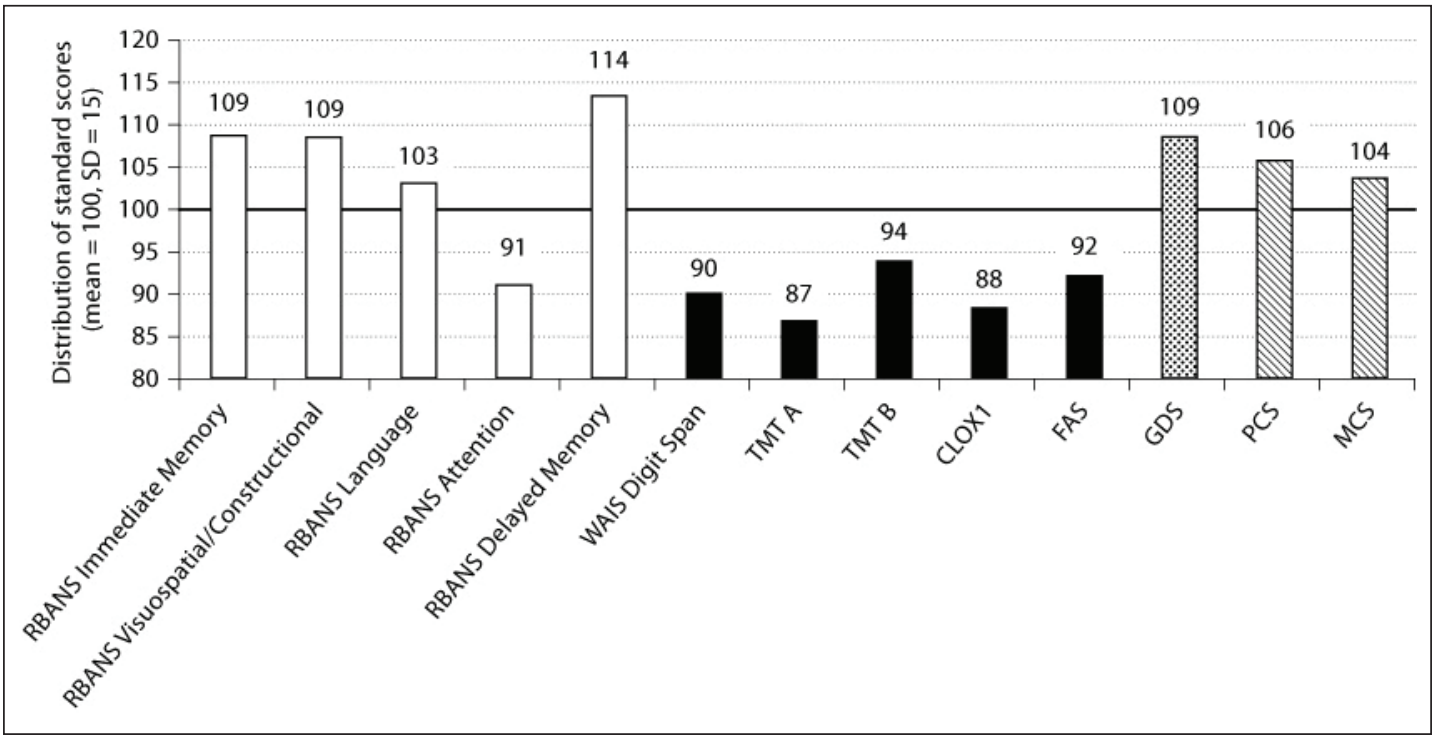

Fig. 1. Cognition, mood, and daily functioning at baseline for an 84-year-old female with 14 years of education.

\section{Discussion}

This article presents results from a small pilot sample selected using specific inclusion/ exclusion criteria for cerebral small vessel disease from a follow-up neurology stroke clinic. These pilot results illustrate some neuropsychological characteristics and imaging findings associated with this disease process. Scores among the older subsample were somewhat lower compared to the younger sample, generally for measures of attention, speed of processing, and memory and executive functioning. In contrast, mood was better among the older group. Performance on a computer-based cognitive measure was generally stable over a short time interval, with a very slight overall decline, which was not significant. Physical functioning showed improvement, possibly associated with recovery from a clinically evident lacunar stroke.

Most participants in this pilot sample demonstrated performance below one standard deviation from the normative mean score in the domains commonly affected by cerebral small vessel disease (i.e. attention, speed, executive functioning, and memory retrieval). For most participants, clinical report findings on SPECT imaging were consistent with cerebral small vessel disease. Mention of small vessel disease in clinical reports for MRI, or notation of white matter hyperintensities or lacunes was mixed for the participants. On structural and functional imaging for the case example with mild neuropsychology findings, the clinical report from structural MRI was normal, while the SPECT report of functional imaging identified perfusion abnormalities.

These preliminary findings are generally consistent with previous studies involving structural and functional imaging. In the prospective Leukoaraiosis and Disability Study [6], white matter hyperintensities and lacunes on MRI were independently associated with general cognitive function in a prospective sample of 639 participants aged 65-84 years. This prospective sample had no or only mild disability in their instrumental activities of daily living. Van Dijk et al. [22] used a semiquantitative scale to rate incident lacunar infarcts and change in periventricular and subcortical white matter lesion severity over a three-year pe- 
riod among 685 persons 60 to 90 years of age. Lesion progression was associated with a parallel decrease in speed of information processing and general cognitive function.

Using SPECT imaging, Kato et al. [23] studied localized regional blood flow changes among 15 participants with early-stage dementia from cerebral small vessel disease. They found hypoperfusion in the pulvinar nucleus of the thalamus compared to controls, and in the left pulvinar thalamic nucleus compared to participants with white matter lesions but without dementia. Shim et al. [24] used SPECT imaging to compare two subtypes of cerebral small vessel disease, Binswanger's disease and the lacunar state. Among 25 participants with cerebral small vessel disease, they found cerebral blood flow reductions in several regions, including the thalamus bilaterally, the anterior cingulated gyri, the superior temporal gyri, the caudate heads, and the left parahippocampal gyrus. The Binswanger's disease suptype did not differ from the lacunar state subtype of cerebral small vessel disease in the pattern of regional cerebral blood flow.

Recent developments in neuroimaging analysis, such as the Easy Z-Score Imaging Analysis System (eZIS), will allow for future research into computer analysis of SPECT images as an adjunct to early diagnosis $[25,26]$. The eZIS analysis allows comparison of an individual SPECT image, or a composite group of images, to a normal database of SPECT images that is adjusted for the specific SPECT camera used to obtain the patient image [27-30]. Specific regions of interest can be examined for hypoperfusion, and perfusion in regions of interest can be associated with neuropsychological test scores through the use of statistical parametric mapping [23, 31].

This descriptive report of preliminary data has a number of limitations. The sample is a small pilot sample without a control group, and so caution is needed in generalizing the results. The comparison between younger and older age groups is only descriptive and should be interpreted as such. The MRI and SPECT data was not reviewed in a blinded fashion and was not scored in a prospective, quantitative manner. A prospective and blind to cognitive status reading using standardized a priori criteria can be used in future studies. Recruitment occurred over an extended time period, as the study was a lower priority than other research studies being conducted at the stroke center, and numerous patients were screened and found ineligible. The sample was collected using strict criteria for cerebral small vessel disease, nonetheless participants had questions of other disease processes raised in the clinical reports of functional and/or structural imaging. One of the participants did not meet criteria for dementia at enrollment, yet performed near or below two standard deviations from the mean on tests of speed, immediate and delayed memory, and verbal fluency. Daily functioning was also in this range. MRI findings were consistent with the cerebral amyloid angiopathy type of small vessel disease. This participant was excluded from the study after the initial assessment.

These results do raise a number of questions for further research. One question involves the potential of functional imaging such as SPECT or fMRI, combined with advances in imaging analysis, to identify the cerebral small vessel disease process even before changes are noticed in cognition. Another question involves whether structural MRI can identify cerebral small vessel disease as well as functional imaging. Cerebral small vessel disease often occurs along with another disease process, such as Alzheimer's disease. Future research can address the differential diagnosis of cerebral small vessel disease as a distinct entity, as well as its role in interaction with other disease entities affecting decline in cognition, mood, and daily functioning. 


\section{Acknowledgements}

Funding for this study was provided by a grant from the Gustavus and Louise Pfeiffer Research Foundation.

We would like to acknowledge the contributions of Kathy Parkes and Cheryl Conover of the Neurology Department of the University at Buffalo and Millard Fillmore Gates Circle Hospital in recruiting patients and coordinating and managing the data collection. We would also like to acknowledge the contributions of the participants to the study.

\section{References}

1 Loeb C, Meyer JS: Vascular dementia: still a debatable entity? J Neurol Sci 1996;143:31-40.

-2 Pantoni L: Cerebral small vessel disease: from pathogenesis and clinical characteristics to therapeutic challenges. Lancet Neurol 2003;9:689-701.

-3 Chui H: Dementia due to subcortical ischemic vascular disease. Clin Cornerstone 2001;3:40-51.

-4 Meyer JS, Xu G, Thornby J, Chowdhury MH, Quach M: Is mild cognitive impairment prodromal for vascular dementia like Alzheimer's disease? Stroke 2002;33:1981-1985.

-5 Grau-Olivares M, Arboix A: Mild cognitive impairment in stroke patients with ischemic cerebral small-vessel disease: a forerunner of vascular dementia? Expert Rev Neurotherap 2009;9:1201-1217.

-6 Jokinen H, Kalska H, Ylikoski R, Madureira S, Verdelho A, van der Flier WM, Scheltens P, Barkhof F, Visser MC, Fazekas F, Schmidt R, O'Brien J, Waldemar G, Wallin A, Chabriat H, Pantoni L, Inzitari D, Erkinjuntti T: Longitudinal cognitive decline in subcortical ischemic vascular disease-the LADIS Study. Cerebrovasc Dis 2009;27:384-391.

-7 Kramer JH, Reed BR, Mungas D, Weiner MW, Chui HC: Executive dysfunction in subcortical ischaemic vascular disease. J Neurol Neurosurg Psychiatry 2002;72:217-220.

-8 Lesage SR, Mosley TH, Wong TY, Szklo M, Knopman D, Catellier DJ, Cole SR, Klein R, Coresh J, Coker LH, Sharrett AR: Retinal microvascular abnormalities and cognitive decline: the ARIC 14year follow-up study. Neurology 2009;73:862-868.

-9 Prins ND, van Dijk EJ, den Heijer T, Vermeer SE, Jolles J, Koudstaal PJ, Hofman A, Breteler MMB: Cerebral small-vessel disease and decline in information processing speed, executive function and memory. Brain 2005;128:2034-2041.

-10 Zhou A, Jia J: Different cognitive profiles between mild cognitive impairment due to cerebral small vessel disease and mild cognitive impairment of Alzheimer's disease origin. J Int Neuropsychol Soc 2009;15:898-905.

-11 Duff K, Patton D, Schoenberg MR, Mold J, Scott JG, Adams RL: Age- and education-corrected independent normative data for the RBANS in a community dwelling elderly sample. Clin Neuropsychol 2003;17:351-366.

-12 Duff K, Schoenberg MR, Patton D, Paulsen JS, Bayless JD, Mold J, Scott JG, Adams RL: Regressionbased formulas for predicting change in RBANS subtests with older adults. Arch Clin Neuropsychol 2005;20:281-290.

-13 Darby D, Maruff P, Collie A, McStephen M: Mild cognitive impairment can be detected by multiple assessments in a single day. Neurology 2002;59:1042-1046.

-14 Maruff P, Thomas E, Cysique L, Brew B, Collie A, Snyder P, Pietrzak RH: Validity of the CogState brief battery: relationship to standardized tests and sensitivity to cognitive impairment in mild traumatic brain injury, schizophrenia, and AIDS dementia complex. Arch Clin Neuropsychol 2009;24: $165-178$.

-15 Sabri O, Hellwig D, Schreckenberger M, Cremerius U, Schneider R, Kaiser HJ, Doherty C, Mull M, Ringelstein EB, Buell U: Correlation of neuropsychological, morphological and functional (regional cerebral blood flow and glucose utilization) findings in cerebral microangiopathy. J Nucl Med 1998; 39:147-154.

-16 Yoshikawa T, Murase K, Oku N, Kitagawa K, Imaizumi M, Takasawa M, Nishikawa T, Matsumoto M, Hatazawa J, Hori M: Statistical image analysis of cerebral blood flow in vascular dementia with small-vessel disease. J Nucl Med 2003;44:505-511. 
-17 Grau-Olivares M, Arboix A, Bartres-Faz D, Junque C: Neuropsychological abnormalities associated with lacunar infarction. J Neurol Sci 2007;257:160-165.

- 18 O’Brien JT, Erkinjuntti T, Reisberg B, Roman G, Sawada T, Pantoni L, Bowler JV, Ballard C, DeCarli C, Gorelick PB, Rockwood K, Burns A, Gauthier S, DeKosky ST: Vascular cognitive impairment. Lancet Neurol 2003;2:89-98.

-19 Roman GC, Erkinjuntti T, Wallin A, Pantoni L, Chui HC: Subcortical ischaemic vascular dementia. Lancet Neurol 2002;1:426-436.

-20 Erkinjuntti T, Inzitari D, Pantoni L, Wallin A, Scheltens P, Rockwood K, Roman GC, Chui H, Desmond DW: Research criteria for subcortical vascular dementia in clinical trials. J Neural Transm 2000;59:23-30.

21 Lezak MD, Howieson DB, Loring DW: Neuropsychological assessment. New York, Oxford University Press, 2004.

-22 van Dijk EJ, Prins ND, Vrooman HA, Hofman A, Koudstaal PJ, Breteler MM: Progression of cerebral small vessel disease in relation to risk factors and cognitive consequences. Stroke 2008;39:2712-2719.

-23 Kato H, Yoshikawa T, Oku N, Imaizumi M, Takasawa M, Kimura Y, Kajimoto K, Tanaka M, Kitagawa K, Hori M, Hatazawa J: Statistical parametric analysis of cerebral blood flow in vascular dementia with small-vessl disease using Tc-HMPAO SPECT. Cerebrovasc Dis 2008;26:556-562.

- 24 Shim YS, Yang DW, Kim BS, Shon YM, Chung YA: Comparison of regional cerebral blood flow in two subsets of subcortical ischemic vascular dementia: statistical parametric mapping analysis of SPECT. J Neurol Sci 2006;250:85-91.

-25 Waragai M, Mizumura S, Yamada T, Matsuda H: Differentiation of early-stage Alzheimer's disease from other types of dementia using brain perfusion single photon emission computed tomography with easy Z-score imagine system analysis. Dement Geriatr Cogn Disord 2008;26:547-555.

-26 Waragai M, Yamada T, Matsuda H: Evaluation of brain perfusion SPECT using an easy Z-score (eZIS) as an adjuct to early-diagnosis of neurodegenerative diseases. J Neurol Sci 2007;260:57-64.

-27 Matsuda H, Mizumura S, Soma T, Takemura N: Conversion of brain SPECT images between different collimators and reconstruction processes for analysis using statistical parametric mapping. Nucl Med Commun 2004;25:67-74.

28 Mizumura S, Kumita S: Stereotactic statistical imaging analysis of the brain using the easy Z-score imaging system for sharing a normal database. Radiat Med 2006;24:545-552.

-29 Matsuda H, Mizumura S, Nagao T, Ota T, Iizuka T, Nemoto K, Kimura M, Tateno A, Ishiwata A, Kuji I, Arai H, Homma A: An easy Z-score imaging system for discrimination between early Alzheimer's disease and controls using brain perfusion SPECT in a multicentre study. Nucl Med Commun 2007; 28:199-205.

-30 Matsuda H, Mizumura S, Nagao T, Ota T, Iizuka T, Nemoto K, Takemura N, Arai H, Homma A: Automated discrimination between very early Alzheimer disease and controls using an easy Z-score imaging system for multicenter brain perfusion single-photon emission tomography. AJNR Am J Neuroradiol 2007;28:731-736.

- 31 Ikeda E, Shiozaki K, Takahashi N, Togo T, Odawara T, Oka T, Inoue T, Hirayasu Y: Total Mini-Mental State Examination score and regional cerebral blood flow using $\mathrm{Z}$ score imaging and automated ROI analysis software in subjects with memory impairment. Ann Nucl Med 2008;22:539-542. 